\title{
On Key Distribution and Authentication in Mobile Radio Networks
}

\author{
Choonsik Park, Kaoru Kurosawa, Tatsuaki Okamoto ${ }^{\dagger}$ and Shigeo Tsujii \\ Department of Electrical and Electronic Engineering, \\ Faculty of Engineering, Tokyo Institute of Technology \\ 2-12-1 O-okayama, Meguro-ku, Tokyo, 152 Japan \\ Email: parkcsoss.titech.ac.jp \\ $\dagger$ NTT Network Information Systems Laboratories, \\ Nippon Telegraph and Telephone Corporation \\ 1-2356, Take, Yokosuka-shi, Kanagawa-ken, 238-03 Japan
}

\begin{abstract}
Mobile communication networks need public key cryptosystems that offer both low computation cost and user authentication. Tatebayashi et al showed such a key distribution protocol for such networks at CRYPTO' 89 based on low exponent RSA. This paper shows that their protocol is not secure. We also present a new secure and efficient key distribution protocol.
\end{abstract}

\section{Introduction}

Security in digital mobile communication systems has two major characteristics that must be be achieved, low computation cost and user authentication. Theoretically, $\mathrm{A}$ (lice) and $\mathrm{B}(\mathrm{ob})$ who have never met can share a cryptographic key by using a public key cryptosystem. The disadvantage of current public key cryptosystems is that encryption and decryption take too long. This disadvantage is serious in mobile communication networks because each user has very small computational power. The user authentication problem is also important to avoid charges of fraudulent usage. Another property of mobile communication networks, which is a good news for us, is that each user communicates with each ather through a network center. Our goal is to design a public key cryptosystem for such networks that realizes both low computation cost and user authentication.

Until recently, however, only slight attention has been paid to this problem. The key distribution protocol shown by Tatebayashi et al.[1] at the 1989 CRYPTO conference is the only product known to the authors.

This paper irst shows that their protocol is not secure. In the protocol of [1], A and $B$ send initial information to the network center by using a low exponent RSA. However, we show that $B$ can find the secret of $A$, needed for user authentication, easily by using this low exponent property. We then present a new key distribution protocol which realizes both low computation cost and user authentication by introducing a simple nonlinear function. 
Our technical contribution is as follows. Hastad [3] showed that we can solve the following simultaneous equations,

$$
\left(\alpha_{i} X+\beta_{i}\right)^{3}=c_{i} \quad\left(\bmod N_{i}\right),(i=1, \cdots, 7),
$$

for $X$ in polynomial time if $\operatorname{gcd}\left(N_{i}, N_{j}\right)=1$ for $i \neq j$ and if the number of equations is seven. Our analysis of [1] shows that we can obtain the following simultaneous equations,

$$
\left(\alpha_{i} X+\beta_{i}\right)^{3}=c_{i} \quad(\bmod N) .
$$

Since $N$ is common for each $i$, Hastad's attack cannot be applied. We show a method for this problem which finds $X$ in polynomial time if the number of equations is three. We propose a key distribution protocol which is secure for both attacks.

o denotes concatenation. $|X|$ denotes the bit length of $X$.

\section{Review of Tatebayashi et al.'s scheme}

The key distribution scheme of [1] was developed in the following process. First, network center $C^{\prime}$ generates an RSA cryptosystem $e=3$. Let

$$
E(M)=M^{3} \bmod N(=p q),
$$

where $N$ is the public key of $C$ and $p, q$ are the secret keys of $C$. Suppose that $A$ and $B$ want to share a key $K$. $X$ and $Y$ are opponents.

\section{$2.1 \mathrm{KDP} 1$}

Their basic protocol KDP1 is as follows.

[KDP1]

1. A chooses a random number $r_{1}$ and computes $Z_{a}=E\left(r_{1}\right)$. $A$ sends $Z_{z}$ to $C$.

2. $B$ chooses a key $K$ randomiy and computes $Z_{b}=E(K)$. $B$ sends $Z_{b}$ to $C$.

3. C' decrypts $Z_{a}$ and $Z_{b}$. It computes $u=r_{1}+K \bmod N$. $C$ send $u$ to $A$.

4. A computes the key $K$ as $K=u-r_{1} \bmod N$.

\subsection{Simmons attack}

Simmons showed one attack on KDP1.

[Simmons' attack]

1. $X$ and $Y$ monitor $Z_{b}$.

2. I chooses a random number $R$ and computes $Z_{x}=E(R)$.

$I$ sends $Z_{x}$ to $C$.

3. $Y$ sends $Z_{b}$ to $C$.

4. $C$ then sends $u^{\prime}=R+K^{\prime} \bmod N$ to $X$.

5. $X$ can compute from $u^{\prime}$ and $R$ the key $K$ of $A$ and $B$. 


\subsection{User authentication}

We should also consider the problem of user anthentication because it is important to avoid charges of fraudulent usage. To eliminate this problem, KDP1 can be modified as follows. Let $f$ be a psendorandom function which is the secret of $C$. Let $I D_{a}$ and $I D_{b}$ be the identity of $A$ and $B$, respectively. In the preprocessing stage, $C$ computes

$$
S_{a}=f\left(I D_{a}\right), \quad S_{b}=f\left(I D_{b}\right)
$$

$C$ sends $S_{a}$ to $A$ secretly. Similarly, $C$ sends $S_{b}$ to $B$ secretly. [KDP1*]

1. A chooses a random number $r_{1}$ and computes $Z_{a}=E\left(S_{a} \circ r_{1}\right)$. $A$ sends $Z_{a}$ and $I D_{a}$ to $C$.

2. $B$ chooses a key $K$ randomly and computes $Z_{b}=E\left(S_{b} \circ K\right)$. $B$ sends $Z_{b}$ and $I D_{b}$ to $C$.

3. $C$ decrypts $Z_{a}$ and $Z_{b}$. It checks that $S_{a}=f\left(I D_{a}\right)$ and $S_{b}=f\left(I D_{b}\right)$. It the check passes, $C$ computes $u=r_{1}+K$ and send $u$ to $A$.

4. A computes the key $K$ as $K=u-r_{1}$.

It is easy to see that Simmons' attack can also be applied to KDP1*.

\section{$2.4 \quad$ KDP2}

Finally, Tatebayashi et al. developed the following protocol KDP2 which uses timestamps to avoid Simmons' attack.

[KDP2]

1. A chooses at random number $r_{1}$ and computes $Z_{a}=E\left(T_{a} \circ S_{a} \circ r_{1}\right)$, where $T_{a}$ is A's timestamp information.

$A$ sends $Z_{a}$ and his identity $I D_{a}$ to the center $C$.

2. $C$ decrypts the ciphertext and verifies the identity of $A$ and the timestamp information. $C$ then calls $B$.

3. $B$ chooses a key $K$ randomly and computes $Z_{b}=E\left(T_{b} \circ S_{b} \circ K\right)$, where $T_{b}$ is $B$ 's timestamp information.

$B$ sends $Z_{b}$ and his identity $I D_{b}$ to the center $C$.

4. $C$ checks $T_{b}$ and $S_{b} . C$ then computes $u=r_{1}+K$ and sends $u$ to $A$.

5. $A$ computes $K^{\prime}$ as $K=u-r_{1}$.

\section{Attack on KDP2}

This section shows that KDP2[1] is not secure. Actually, we show that $B$ can find $A$ 's secret information $S_{a}$ if $B$ executes KDP2 with $A$ three times.

After repeating KDP2 three times, $B$ obtains

$$
Z_{a i}=E\left(T_{a i} \circ S_{a} \circ r_{1 i}\right), \quad u_{i}=r_{1 i}+K_{i}, \quad(i=1,2,3)
$$

by listening to the conversation between $A$ and the center $C$. $B$ then gets 
$-r_{1 i}$ from $u_{i}$ and $K_{i}$.

- $T_{a i}$ because it is the time at which $A$ sends $Z_{a i}$ to $C$.

The unknown constant is only $S_{a}$. Suppose that

$$
\left|r_{1 i}\right|=1, \quad\left|S_{a}\right|+\left|r_{1 i}\right|=m
$$

$Z_{a i}$ is written as follows.

$$
\begin{aligned}
& \left(T_{a 1} \times 2^{m}+S_{a} \times 2^{l}+r_{11}\right)^{3}=Z_{a 1}(\bmod N) \\
& \left(T_{a 2} \times 2^{m}+S_{a} \times 2^{l}+r_{12}\right)^{3}=Z_{a 2}(\bmod N) \\
& \left(T_{a 3} \times 2^{m}+S_{a} \times 2^{l}+r_{13}\right)^{3}=Z_{a 3}(\bmod N)
\end{aligned}
$$

Let $X_{a}=S_{n} \times 2^{\prime}$ and $Y_{i}=T_{n i} \times 2^{m}+r_{1 i}$. We then obtain

$$
\begin{aligned}
& X_{a}^{-3}+3 X_{a}^{2} \times Y_{1}+3 \times\left(Y_{1}\right)^{2} \times X_{a}=Z_{a 1}-\left(Y_{1}\right)^{3}(\bmod N), \\
& X_{a}^{3}+3 X_{a}^{2} \times Y_{2}+3 \times\left(Y_{2}\right)^{2} \times X_{a}=Z_{a 2}-\left(Y_{2}\right)^{3}(\bmod N), \\
& X_{a}^{3}+3 X_{a}^{2} \times Y_{3}+3 \times\left(Y_{3}\right)^{2} \times X_{a}=Z_{a 3}-\left(Y_{3}\right)^{3}(\bmod N) .
\end{aligned}
$$

The only unknown constant is $X_{a}$. We can view the above equations as linear simultaneous equations on $X_{a}^{-3}, X_{a}^{-2}$ and $X_{a} . B$ can easily solve these equations in polynomial time. $B$ can compute $S_{a}$ from the solution $X_{n}$.

This attack works even if $T_{n}, S_{a}$ and $r_{1}$ are interleaved. It also works for $E(M)=M^{e} \bmod N$ if $e$ is small.

Similarly, $A$ can find the secret information $S_{b}$ of $B$ because KDP2 is symmetric for $A$ and $B$.

\section{Proposed scheme}

The reason why our attack succeeds is that $B$ can obtain $r_{1 i}$ ( $A$ can obtain $K_{i}$ ) from the equation

$$
u_{i}=r_{1 i}+I_{i} \text {. }
$$

We can prevent our attack if we introduce a nonlinear function $h$ and modify eq. (1) as Eollows.

$$
u_{i}=h\left(r_{1 i}\right)+h\left(K_{i}\right) .
$$

For example, the following simple $h$ is enough.

$$
h\left(x_{1} \circ x_{2}\right)=x_{1}+x_{2} \bmod 2^{l / 2}
$$

where $\left|x_{1}\right|=\left|x_{2}\right|=1 / 2$ and 0 denotes concatenation.

It is information theoretically impossible to determine $x_{1}$ and $x_{2}$ from $x_{1}+x_{2}$. Based on this observation, we propose a key distribution protocol as follows.

( $E$ is the public key of the center. See the beginning of section 2.)

1. A chooses random numbers $\left(r_{1}, r_{2}\right)$ such that $\left|r_{1}\right|=\left|r_{2}\right|=1 / 2$ and computes $Z_{a}=E\left(T_{a} \circ S_{a} \circ r_{1} \circ r_{2}\right)$, where $T_{a}$ is $A$ 's timestamp information. $A$ sends $Z_{a}$ and his identity $I D_{a}$ to the center $C$. 
2. $C$ decrypts the ciphertext and verifies the identity of $A$ and the timestamp information. $C$ then calls $B$.

3. $B$ chooses $\left(K_{1}, K_{2}\right)$ randomly and computes $Z_{b}=E\left(T_{b} \circ S_{b} \circ K_{1} \circ K_{2}\right)$, where $T_{b}$ is $B$ 's timestamp information.

$B$ sends $Z_{b}$ and his jdentity $I D_{h}$ to the center $C$.

4. $C$ checks $T_{b}$ and $S_{b} . C$ then computes $u=r_{1}+r_{2}+K_{1}+K_{2} \bmod 2^{1 / 2}$ and send $u$ to $A$.

5. The session key is given by $K=K_{1}+K_{2} \bmod 2^{1 / 2}$. A computes $K$ as $K=u-r_{1}-r_{2} \bmod 2^{l / 2}$.

We can use the Rabin cryptosystem instead of RSA because the plaintext has a special data structure.

\section{Security}

Suppose that the proposed protocol is executed $I$ times. Let the $i$ th parameters be $Z_{2 i}, T_{a i}, r_{1 i}, r_{2 i}, Z_{b i}, T_{b i}, K_{1 i}$ and $K_{2 i} . B$ knows that

$$
Z_{a i}=E\left(T_{a i} \circ S_{a} \circ r_{1 i} \circ r_{2 i}\right) \text {. }
$$

He knows the values of $Z_{a i}, T_{a i}$ and $r_{1 i}+r_{2 i} \quad\left(\bmod 2^{i / 2}\right)$ by monitoring $A$ 's communication. However, he can not know $r_{1 i} \circ r_{2 i}$. (o denotes concatenation.) Therefore, what $B$ can have is the following type of equations,

$$
\left(\alpha_{i} X+Y_{i}+\beta_{i}\right)^{3}=c_{i} \quad(\bmod N),(i=1, \cdots, I),
$$

where $X=S_{a}, Y_{i}=r_{1 i} \circ r_{2 i}, \alpha_{i}=2^{\prime}, \beta_{i}=T_{n i} \times 2^{\left(l+\left|S_{a}\right|\right)}, c_{i}=Z_{a i}$. Here, $X$ and $Y_{i}$ are unknown variables. $\alpha_{i}, \beta_{i}$ and $c_{i}$ are known values.

Then, the number of equations is $I$ and that of unknown variables is $I+1$. Hence, $B$ cannot find $X\left(=S_{a}\right)$. Similarly, A cannot find $S_{b}$.

\section{References}

1. Tatebayashi, MI., Matsuzaki, N., Newman, Jr., D.B.: Key Distribution Protocol for Digital Mobile Communication Systems. Advances in Cryptology: Proceedings of Crypto'89 (1989) 324-334

2. Moore, J.H.: Protocol Failures in Cryptosystems. Proc of IEEE, Vol.76, No.5 (1988) $594-602$

3. Hastad,J.: On using RSA with Low exponent in a public key network. Advances in Cryptology, Proceedings of Crypto's5 (1985)403-408

4. Simmons, G.J.: A 'weak' privacy protocol using the RSA cryptoalgorithm. Cryptolo. gia, Vol.t (1983) 180-182

5. Beller,M.J. Chang,L.F., Yacobi,Y. : Privary and Authentication on a Portable Communication System. IEEE GLOBECOW '91 conference (1991) 1922-1927 